\title{
Whole-Body Vibration Sensor Calibration Using a Six-Degree of Freedom Robot
}

\author{
Sarah Cation, ${ }^{1}$ Michele Oliver, ${ }^{1,2}$ Robert Joel Jack, ${ }^{1,2,3}$ \\ James P. Dickey, ${ }^{2,4}$ and Natasha Lee Shee ${ }^{2}$ \\ ${ }^{1}$ School of Engineering, University of Guelph, Guelph, ON, Canada N1G 2W1 \\ ${ }^{2}$ Biophysics Interdepartmental Group Graduate Program, University of Guelph, Guelph, ON, Canada N1G 2W1 \\ ${ }^{3}$ School of Human Kinetics, Laurentian University, Sudbury, ON, Canada P3E 2C6 \\ ${ }^{4}$ School of Kinesiology, University of Western Ontario, London, ON, Canada N6A 3K7 \\ Correspondence should be addressed to Michele Oliver, moliver@uoguelph.ca
}

Received 23 September 2010; Revised 7 February 2011; Accepted 8 March 2011

Academic Editor: Gurvinder Virk

Copyright () 2011 Sarah Cation et al. This is an open access article distributed under the Creative Commons Attribution License, which permits unrestricted use, distribution, and reproduction in any medium, provided the original work is properly cited.

\begin{abstract}
Exposure to whole-body vibration (WBV) is associated with a wide variety of health disorders and as a result WBV levels are frequently assessed. Literature outlining WBV accelerations rarely address the calibration techniques and procedures used for WBV sensors to any depth, nor are any detailed information provided regarding such procedures or sensor calibration ranges. The purpose of this paper is to describe a calibration method for a 6 DOF transducer using a hexapod robot. Also described is a separate motion capture technique used to verify the calibration for acceleration values obtained which were outside the robot calibration range in order to include an acceptable calibration range for WBV environments. The sensor calibrated in this study used linear $(Y=m X)$ calibration equations resulting in $r^{2}$ values greater than 0.97 for maximum and minimum acceleration amplitudes of up to $\pm 8 \mathrm{~m} / \mathrm{s}^{2}$ and maximum and minimum velocity amplitudes up to $\pm 100^{\circ} / \mathrm{s}$. The motion capture technique verified that the translational calibrations held for accelerations up to $\pm 4 \mathrm{~g}$. Thus, the calibration procedures were shown to calibrate the sensor through the expected range for 6-DOF WBV field measurements for off-road vehicles even when subjected to shocks as a result of high speed travel over rough terrain.
\end{abstract}

\section{Introduction}

With technological advancements, the detail and complexity of the measurements researchers are able to undertake has increased. This is exemplified in the field of whole-body vibration (WBV). The most recent publication of the ISO 2631-1 standard outlines the measurement of WBV in 6 degrees-offreedom (DOF), and some researchers have suggested that 12 DOF measurements should be performed [1]. Thus, measurements in translational axes $X-, Y$-, and $Z$ - as well as rotational axes roll, pitch, and yaw (about $X-, Y_{-}$, and $Z$-axes, resp.) are required. Exposure to excessive levels of whole-body vibration is associated with decreased worker performance and injury [2]; the vibration limits described in the International and British standards ([3] and BS 6841 [4], resp.) and European Directive 2002/44/EC, are important benchmarks for assessing human exposure to whole-body vibration [5]. Compliance with the ISO and BS standards is recommended for reducing the risk of workplace injury. In contrast, the European Directive mandates that health surveillance is compulsory for workers exposed to vibration levels that exceed the daily exposure action values. This progression from assessment to compulsory surveillance has motivated increased vibration measurements in the workplace. The movement to 6-DOF and 12-DOF measurements has increased the complexity of the sensors used to measure WBV.

In some WBV environments, there may be transients (or shocks), which by definition are sudden changes in accelerations [2]. Off-road vehicles are likely often subjected to shocks as a result of high speeds over rough terrain $[6,7]$. Based on forestry skidder WBV literature, the WBV transducer is required to record peak accelerations of no less than $7.08 \pm 3.48 \mathrm{~m} / \mathrm{s}^{2}[6]$ or no less than $3.32 \pm 1.42 \mathrm{~m} / \mathrm{s}^{2}[8]$ at 
the operator seat interface (OSI) in the translational axes. The reported dominant frequency band was $2.5 \mathrm{~Hz}$ at the chassis [9]. In contrast, accelerations at the OSI of up to $11.73 \mathrm{~m} / \mathrm{s}^{2}$ have been reported for heavy haul dump trucks [10]. These published values have been weighted according to various versions of the ISO 2631-1 standard. Accordingly, since the ISO 2631 frequency weightings attenuate the acceleration signals according to the sensitivity to vibration at various frequencies, it is therefore important to calibrate the WBV sensors for a larger amplitude range than reported in these papers. This should be done to ensure that there has been no underestimation of the actual recorded field accelerations which may be attenuated at certain frequencies during the weighting process.

In the field of WBV, the literature outlining WBV accelerations rarely address the calibration technique/procedures used for WBV sensors to any depth, nor is any detailed information provided regarding such procedures or sensor calibration ranges. Therefore, the purpose of this paper was to describe a simple calibration method for a 6 DOF OSI transducer used for WBV measurements. Also described is a separate technique used to verify the calibration for acceleration values obtained which were outside the robot calibration range in order to include an acceptable calibration range for WBV environments.

\section{Methodology}

A seat-pad transducer (SPT) used for WBV measurements at the OSI was calibrated in two phases. The first calibration phase used a 6-DOF robot to generate the calibration equations for each DOF. The second phase was used to confirm that the robot determined calibration equations would hold outside of their calibrated range. This larger acceleration range was designed to represent the range of vibrations observed in forestry skidders. The SPT was designed to be compact and flat to remove pressure points as it was to be used at the OSI in the field to measure WBV. The sensor was built at the University of Guelph (Guelph, Ontario, Canada) using two Analog Devices (Analog Devices Inc., Norwood, Massachusetts, USA) ADXL320EB biaxial accelerometers measuring the $X, Y$, and $Z$ axes and three Analog Devices ADXRS150EB rate gyroscopes to measure roll, pitch, and yaw. The ADXL320EB accelerometers were rated for $\pm 5 \mathrm{~g}$ $( \pm 10 \%)$ and the ADXRS150EB gyroscopes were capable of recording $\pm 150^{\circ} / \mathrm{s}$ velocities. The accelerometers and gyroscopes were mounted to evaluation boards to simplify mechanical attachment, relative alignment and wiring. The accelerometers and rate gyroscopes were orthogonally placed in a Delrin plastic casing to monitor the roll, pitch, and yaw axes. For both calibration phases, a 12-bit SOMAT Series 2001 Field computer (nCode International Inc., Southfield, Michigan, USA) collected raw device voltages at a sampling rate of $500 \mathrm{~Hz}$.

2.1. Experiment Setup. The first phase was the low $g$ (LG) calibration (maximum and minimum amplitude values of: \pm 1 to $\pm 8 \mathrm{~m} / \mathrm{s}^{2}$ and \pm 10 to $\pm 100^{\circ} / \mathrm{s}$ ) to determine the calibration

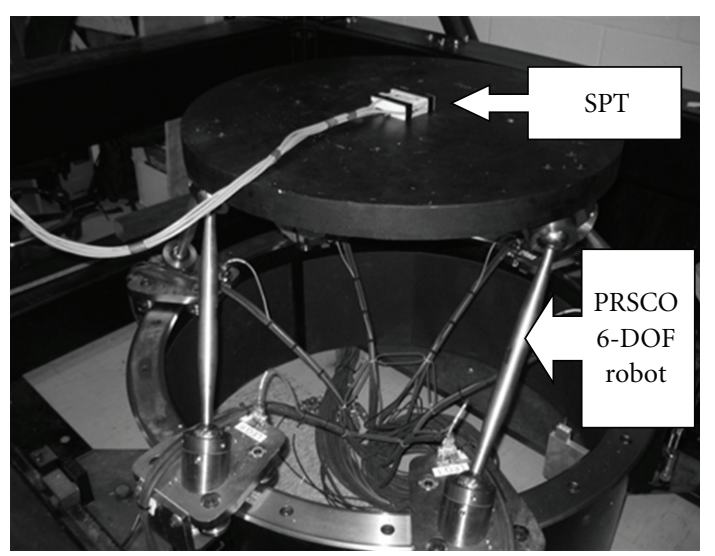

FIgURE 1: Experiment Setup for the low g (LG) calibration using a Parallel Robotics System Corporation (PRSCO) six-degree-offreedom (6-DOF) robot shown here with the seat-pad transducer (SPT).

equations using a 6-DOF robot. The second phase was used to assess the sensor calibration previously determined in the LG calibration using an expanded range of WBV values in order to validate the calibration values determined in the LG calibration. The range consisted of the expected field range for WBV measurements of forestry skidders. The high g (HG) calibration used a vibration table and an optical motion capture system. The sensor was zeroed before each axis was tested in order to eliminate orientation bias.

2.1.1. Low $g(L G)$ Calibration. The LG calibration was completed using a Parallel Robotics System Corporation (PRSCO) R2000 6-DOF robot (Parallel Robotic Systems Corporation, Hampton, New Hampshire, USA). The PRSCO was calibrated by PRSCO for repeatability and accuracy (within $24 \mu \mathrm{m}$ ), and accuracy (within $+24 /-38 \mu \mathrm{m}$ ) for travel in the $X, Y$, and $Z$ axes. Sinusoidal acceleration and velocity profiles were created as inputs to the PRSCO. The acceleration profiles were developed to achieve acceleration amplitudes between \pm 1 and $\pm 8 \mathrm{~m} / \mathrm{s}^{2}$ for the translational axes $(X, Y, Z)$ at known frequencies (Table 1$)$ and were double integrated using the trapezoid rule prior to implementation on the robot. The profiles were integrated into displacement form as the robot requires displacement inputs. For pitch, roll, and yaw, a single integration was required, since the sensors for these axes monitor angular velocity. Angular velocity profiles ranged between \pm 10 and $\pm 100^{\circ} / \mathrm{s}$ at frequencies between 0.5 and $5 \mathrm{~Hz}$ as shown in Table 2 . The sensors were placed in the centre of the PRSCO 6DOF robot platform which reproduced the known displacement profiles. The sensors were oriented such that the axis of interest was aligned such that the PRSCO robot would have the largest movement displacement allowing higher accelerations and angular velocities to be produced. The experimental Setup is shown in Figure 1.

2.1.2. High $g(H G)$ Calibration. The LG calibration calibrated up to $\pm 100^{\circ} / \mathrm{s}$, which exceeded the expected field 
TABLe 1: The sinusoidal minimum and maximum linear acceleration amplitudes and corresponding frequencies used to calibrate the translational $(X, Y, Z)$ accelerometers using displacement profiles on a Parallel Robotics System Corporation Robot six-degree-of-freedom robot.

\begin{tabular}{|c|c|c|c|c|c|c|c|c|}
\hline Acceleration $\left(\mathrm{m} / \mathrm{s}^{2}\right)$ & \pm 1 & \pm 2 & \pm 3 & \pm 4 & \pm 5 & \pm 6 & \pm 7 & \pm 8 \\
\hline Frequency $(\mathrm{Hz})$ & $1,2,5$ & $1,2,5$ & 2,5 & 2,5 & 2,5 & 2,5 & 2,5 & 2,5 \\
\hline
\end{tabular}

TAвLE 2: The sinusoidal minimum and maximum angular velocity amplitudes and corresponding frequencies used to calibrate the gyroscopes (roll, pitch, yaw) using displacement profiles on a Parallel Robotics System Corporation Robot six-degree-of-freedom robot.

\begin{tabular}{|c|c|c|c|c|c|c|c|}
\hline Velocity $(\% / s)$ & \pm 10 & \pm 20 & \pm 30 & \pm 40 & \pm 60 & \pm 80 & \pm 100 \\
\hline Frequency $(\mathrm{Hz})$ & $0.5,1,2,5$ & $0.5,1,2,5$ & $0.5,1,2,5$ & $0.5,1,2,5$ & $0.5,1,2,5$ & $0.5,1,2,5$ & $0.5,1,2$ \\
\hline
\end{tabular}

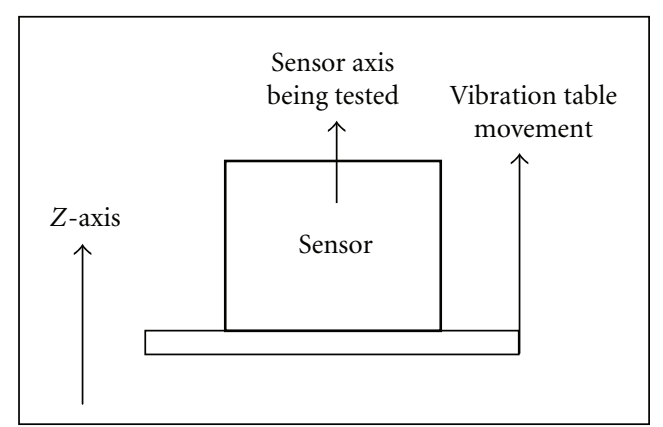

Figure 2: Sensor axis orientation and vibration direction for the high g calibration verification.

requirements for the gyroscopes, but did not encompass the entire expected field requirements for the accelerometers. Thus, a second stage was completed to calibrate the accelerometers up to and including approximately $\pm 4 \mathrm{~g}$ minimum and maximum acceleration amplitudes at frequencies between 10 and $35 \mathrm{~Hz}$.

An All American vibration fatigue testing machine (VTM) (All American Tool \& Mfg. Co., Illinois, USA) was used to calibrate at HGs. This vibration machine is only able to move in the $Z$-axis, thus the axis being tested was oriented with respect to the $Z$ axis for each of the accelerometer axes as shown in Figure 2. The SPT movement on the VTM was recorded using three VICON $\mathrm{M}^{2} \mathrm{~m}$ cameras from a VICON 460 Motion Capture System (VICON Motion Systems, Centennial, CO, USA) and simultaneous data were recorded from the SPT. The experimental Setup is shown in Figure 3. The various accelerations were tested by changing the VTM using an analog frequency setting dial (between approximately $10 \mathrm{~Hz}$ and $35 \mathrm{~Hz}$ ) and peak-topeak displacements (between approximately $1.27 \mathrm{~mm}$ to $5.08 \mathrm{~mm}$ ) outlined in Table 3. The accelerations tested for HG calibration verification included and exceeded the accelerations determined at the LG accelerations which were used to generate the calibration equations. The VICON 460 $\mathrm{M}^{2} \mathrm{~m}$ cameras were previously validated for vibration measurements of displacements greater than $1 \mathrm{~mm}$ to contain less than $5 \%$ error for frequencies between 3 and $30 \mathrm{~Hz}$ [11].

Four, $14 \mathrm{~mm}$ diameter spherical reflective markers were adhered to the sensor/vibration table using double-sided

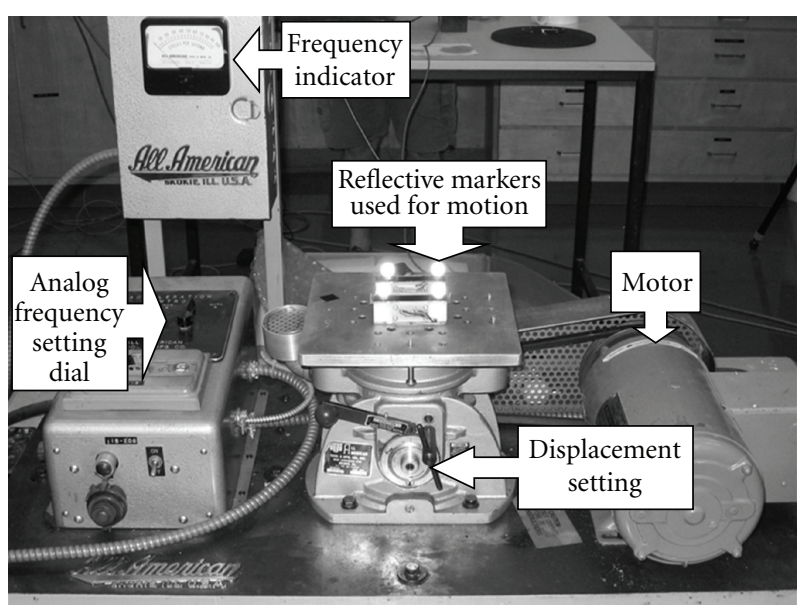

FIGURE 3: The experimental Setup for the high g (HG) calibration verification.

TABLE 3: The peak-to-peak displacements and frequency settings used for the high g calibration verification and resulting \pm approximate peak acceleration amplitude values.

\begin{tabular}{lcc}
\hline $\begin{array}{l}\text { Peak-to-peak displacement } \\
(\mathrm{mm})\end{array}$ & $\begin{array}{c}\text { Frequency } \\
(\mathrm{Hz})\end{array}$ & $\begin{array}{c}\text { Approximate } \\
\text { acceleration }(\mathrm{g})\end{array}$ \\
\hline 5.08 & 10 & 1.02 \\
1.27 & 20 & 1.01 \\
3.81 & 15 & 1.72 \\
2.54 & 20 & 2.03 \\
3.81 & 20 & 3.04 \\
2.54 & 25 & 3.17 \\
1.27 & 35 & 3.08 \\
5.08 & 20 & 4.06 \\
\hline
\end{tabular}

tape such that all $\mathrm{M}^{2} \mathrm{~m}$ cameras had full view of the markers. The cameras were oriented in an umbrella fashion (Figure 4) often used in gait analysis [12]. The cameras recorded the $X, Y$, and $Z$ lab coordinates of the reflective markers for each trial and were sampled at $500 \mathrm{~Hz}$ for 10 seconds. The VICON system was calibrated statically and dynamically in the measurement volume to minimize error due to the optical distortion phenomena [13]. The calibration residuals 


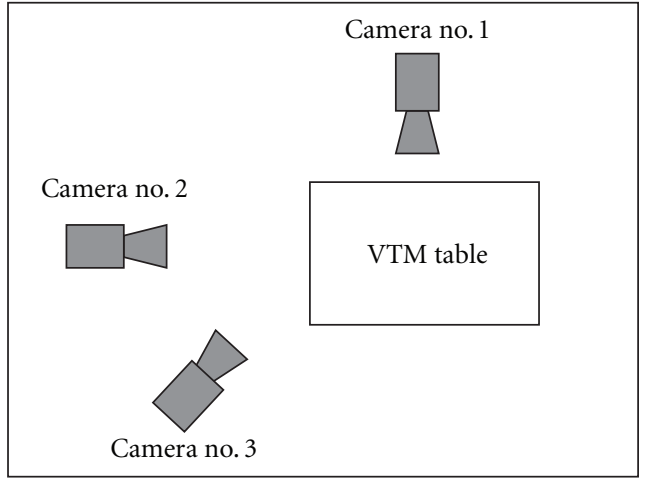

Figure 4: The umbrella camera orientation.

(error) were less than $1 \mathrm{~mm}$ during the calibration of the VICON motion capture system.

\subsection{Data Analysis}

2.2.1. Calibration at Low $g$ Values. The raw dynamic calibration data from the SPT were then second-order low pass Butterworth filtered at a cut-off frequency of $10 \mathrm{~Hz}$ (two times the highest expected signal frequency of $5 \mathrm{~Hz}$ as shown in Tables 1 and 2) such that the Nyquist rate was exceeded. The acceleration and velocity known input profiles were then aligned with the sensor voltage output for the translational and rotational axes, respectively. The calibration equations were determined for each sensor and each axis independently. The calibration equations were determined to be of the form $Y=m X$ for all 6-DOF for the SPT, where $Y$ refers to the calibrated output and $X$ refers to the recorded voltage. The zero offset values are not presented, since they are easily identified and calculated from the resting transducer voltages.

\subsubsection{Translational Acceleration Data Validation at High $g$} $(H G)$ Values. The sensor voltages and raw digitized VICON $3 \mathrm{D}$ coordinates were second-order zero lag low pass Butterworth filtered at a cut-off frequency of two times the frequency set on the VTM. The calibrations determined from the LG calibration were applied to the filtered sensor voltages and then compared to the double-differentiated, filtered VICON displacement data for the three translational axes $(X$, $Y, Z)$. Five individual cycles of double-differentiated VICON displacement data and sensor data were manually aligned using peak acceleration values. Maximum and minimum acceleration amplitudes as well as $r^{2}$ values were determined from the VICON and SPT data for five individual cycles at each VTM setting outlined in Table 3. In addition, the corresponding percent error was determined for the difference between the sensor maximum and minimum acceleration amplitude and VICON maximum and minimum acceleration amplitude, where the VICON data were taken to be the gold standard. The overall average $(n=5)$ was reported using the five single cycle values. This was completed for each of the VTM settings listed in Table 3.
TABle 4: Calibration equations for the seat-pad transducer developed using a low $g$ acceleration values where $Y=m X+b$; slope values $m$ are tabulated, $Y$ refers to the calibrated output and $X$ refers to the recorded voltage.

\begin{tabular}{llc}
\hline Axis & Calibration equation & Unit of measurement \\
\hline$X$ & $Y=-52.02 X$ & $\mathrm{~m} / \mathrm{s}^{2}$ \\
$Y$ & $Y=-52.18 X$ & $\mathrm{~m} / \mathrm{s}^{2}$ \\
$Z$ & $Y=51.13 X$ & $\mathrm{~m} / \mathrm{s}^{2}$ \\
Roll & $Y=81.47 X$ & $\% / \mathrm{s}$ \\
Pitch & $Y=-84.34 X$ & $\% / \mathrm{s}$ \\
Yaw & $Y=79.22 X$ & $\% / \mathrm{s}$ \\
\hline
\end{tabular}

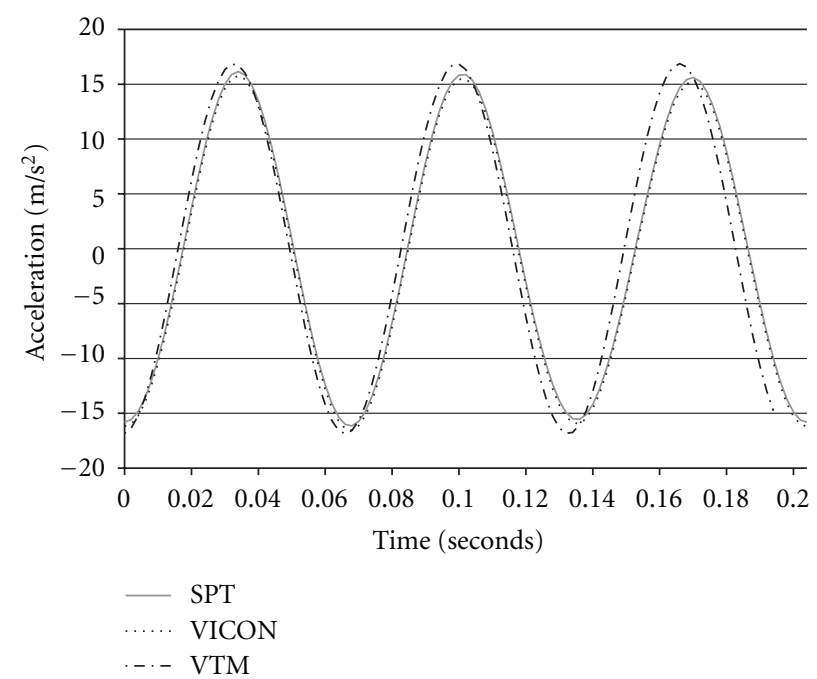

FIGURE 5: The expected acceleration profile is shown (VTM) alongside the accelerations determined using the seat-pad transducer (SPT) and VICON for a high g test with a peak-to-peak displacement of $3.81 \mathrm{~mm}$ and frequency setting of $15 \mathrm{~Hz}$.

\section{Results}

The SPT calibration equations are shown in Table 4. The calibration equations fit the LG calibration data with $r^{2}$ values of greater than 0.997 in all 6-DOF. When the LG calibration equations were applied to HG data and compared to VICON determined accelerations, the accuracy of the sensor was shown to generally produce less than $10 \%$ error. The largest error between the VICON and the system gold standard SPT data was approximately $12 \%$ (under $6 \mathrm{~m} / \mathrm{s}^{2}$ ). This error was less than $12 \%$ at both the maximum and minimum peaks. For the largest acceleration values, the SPT recorded acceleration amplitudes of approximately $\pm 45.63 \mathrm{~m} / \mathrm{s}^{2}$ and VICON predicted accelerations of $\pm 51.58 \mathrm{~m} / \mathrm{s}^{2}$. The $r^{2}$ values exceeded 0.9 in all translational axes at all acceleration levels tested at the HG levels as shown in Table 5.

The effects of using VICON in addition to the known VTM settings were visually assessed, an example of which is shown in Figure 5. As can be seen in the figure, there is a shift between the VTM and the SPT which is not constant over time. In contrast, the SPT and VICON data are well aligned. 
TABLE 5: Results of high g acceleration data verification for the seat-operator interface (SPT) transducer for the $X, Y$, and $Z$ axes at various displacement and frequency settings. The reported accelerations are maximum and minimum amplitudes while the displacements are peakto-peak values.

\begin{tabular}{|c|c|c|c|c|c|c|c|c|}
\hline \multicolumn{9}{|l|}{$\bar{X}$} \\
\hline Displacement $(\mathrm{mm})$ & 1.27 & 5.08 & 2.54 & 3.81 & 1.27 & 2.54 & 3.81 & 5.08 \\
\hline Frequency $(\mathrm{Hz})$ & 20 & 10 & 20 & 15 & 35 & 25 & 20 & 20 \\
\hline$r^{2}$ & 0.97 & 0.98 & 0.95 & 0.96 & 0.92 & 0.97 & 0.95 & 0.98 \\
\hline Maximum camera acceleration $\left(\mathrm{m} / \mathrm{s}^{2}\right)$ & 11.48 & 8.40 & 17.65 & 15.74 & 28.80 & 25.23 & 25.82 & 39.79 \\
\hline Maximum sensor acceleration $\left(\mathrm{m} / \mathrm{s}^{2}\right)$ & 10.37 & 8.74 & 16.93 & 15.93 & 28.96 & 25.09 & 26.75 & 39.17 \\
\hline Maximum acceleration percent error (\%) & 9.61 & 4.01 & 4.01 & 3.21 & 3.81 & 2.62 & 3.62 & 2.95 \\
\hline Minimum camera acceleration $\left(\mathrm{m} / \mathrm{s}^{2}\right)$ & -11.48 & -8.40 & -17.65 & -16.44 & -28.80 & -25.23 & -25.82 & -40.73 \\
\hline Minimum sensor acceleration $\left(\mathrm{m} / \mathrm{s}^{2}\right)$ & -10.37 & -9.05 & -16.93 & -16.02 & -28.96 & -25.09 & -26.50 & -39.17 \\
\hline Minimum acceleration percent error (\%) & 9.61 & 7.77 & 4.01 & 2.54 & 3.81 & 2.62 & 3.30 & 3.77 \\
\hline \multicolumn{9}{|l|}{$\bar{Y}$} \\
\hline Displacement $(\mathrm{mm})$ & 1.27 & 5.08 & 2.54 & 3.81 & 1.27 & 2.54 & 3.81 & 5.08 \\
\hline Frequency $(\mathrm{Hz})$ & 20 & 10 & 20 & 15 & 35 & 25 & 20 & 20 \\
\hline$r^{2}$ & 0.98 & 0.99 & 0.92 & 0.96 & 0.95 & 0.96 & 0.98 & 0.99 \\
\hline Maximum camera acceleration $\left(\mathrm{m} / \mathrm{s}^{2}\right)$ & 10.42 & 9.90 & 20.33 & 15.38 & 29.49 & 32.30 & 27.88 & 40.40 \\
\hline Maximum sensor acceleration $\left(\mathrm{m} / \mathrm{s}^{2}\right)$ & 10.54 & 9.47 & 18.76 & 17.00 & 29.00 & 29.06 & 28.21 & 36.69 \\
\hline Maximum acceleration percent error (\%) & 4.53 & 4.42 & 7.72 & 10.55 & 1.61 & 9.99 & 2.13 & 9.16 \\
\hline Minimum camera acceleration $\left(\mathrm{m} / \mathrm{s}^{2}\right)$ & -10.42 & -9.90 & -20.33 & -15.48 & -29.49 & -32.30 & -27.88 & -40.40 \\
\hline Minimum sensor acceleration $\left(\mathrm{m} / \mathrm{s}^{2}\right)$ & -10.54 & -9.58 & -18.76 & -17.18 & -29.00 & -29.06 & -28.21 & -36.69 \\
\hline Minimum acceleration percent error (\%) & 4.53 & 3.28 & 7.72 & 10.97 & 1.61 & 9.99 & 2.13 & 9.16 \\
\hline \multicolumn{9}{|l|}{$Z$} \\
\hline Displacement $(\mathrm{mm})$ & 1.27 & 5.08 & 2.54 & 3.81 & 1.27 & 2.54 & 3.81 & 5.08 \\
\hline Frequency $(\mathrm{Hz})$ & 20 & 10 & 20 & 15 & 35 & 25 & 20 & 20 \\
\hline$r^{2}$ & 0.92 & 1.00 & 0.99 & 0.99 & 0.98 & 0.94 & 0.99 & 0.98 \\
\hline Maximum camera acceleration $\left(\mathrm{m} / \mathrm{s}^{2}\right)$ & 12.07 & 8.28 & 20.10 & 17.90 & 51.58 & 29.18 & 27.35 & 36.05 \\
\hline Maximum sensor acceleration $\left(\mathrm{m} / \mathrm{s}^{2}\right)$ & 11.54 & 8.15 & 19.08 & 16.36 & 45.63 & 30.48 & 26.51 & 33.23 \\
\hline Maximum acceleration percent error (\%) & 4.37 & 4.20 & 5.07 & 7.09 & 11.46 & 4.77 & 3.07 & 7.82 \\
\hline Minimum camera acceleration $\left(\mathrm{m} / \mathrm{s}^{2}\right)$ & -12.07 & -8.29 & -20.10 & -18.24 & -51.58 & -29.18 & -27.35 & -36.05 \\
\hline Minimum sensor acceleration $\left(\mathrm{m} / \mathrm{s}^{2}\right)$ & -11.54 & -8.32 & -19.08 & -16.85 & -45.63 & -30.48 & -26.51 & -33.23 \\
\hline Minimum acceleration percent error (\%) & 4.37 & 5.01 & 5.07 & 7.62 & 11.46 & 4.77 & 3.07 & 7.82 \\
\hline
\end{tabular}

There was minimal cross-talk encountered in the SPT (Figure 6). Cross-talk was determined by expressing the rms of the individual axes as a percent of the rms of the vibration axis (mean $\pm \mathrm{SD}$ ). For the representative data presented in Figure 6, cross-talk for the translational vibration axes was $7.7 \pm 2.5 \%$, while for the rotational vibration axes, cross-talk was $1.7 \pm 0.6 \%$.

\section{Discussion}

The LG calibration method produced near perfect calibrations which held throughout the expected field range as shown through the HG data verification (Table 5). The errors encountered in the LG and HG acceleration values showed no apparent trends based on frequency or displacement (Table 5). The errors shown in Table 5 were based on the VICON double-differentiated accelerations as it appeared that the true acceleration was different than the dial settings of the VTM (Figure 5) to introduce a phase lag between the VTM and the VICON and SPT accelerations. In the interest of keeping the required calibration time minimal, the VTM was not calibrated. The calibration method being developed is more widely applicable when using any vibration table alongside a calibrated optical measurement system. Because the VTM was not calibrated, the accuracy of analog frequency settings and displacements were not validated. This is likely the reason for part of the shift between the VTM and SPT data shown in Figure 5. The VTM data (Figure 5) were determined using the manual settings (peak-to-peak displacement and frequency) to determine the expected acceleration of the VTM. This is also why the VTM data were not included in the calibration verification process and why the VICON Motion Capture System was used.

The VICON motion capture system is not without issues. It has been noted that any measurement error in 3-D marker coordinates may propagate unpredictably [13]. For example, the double differentiation may have also introduced high frequency errors which result from small errors in displacement data [14]. The data were low pass filtered so it is believed any error resulting from the double differentiation 


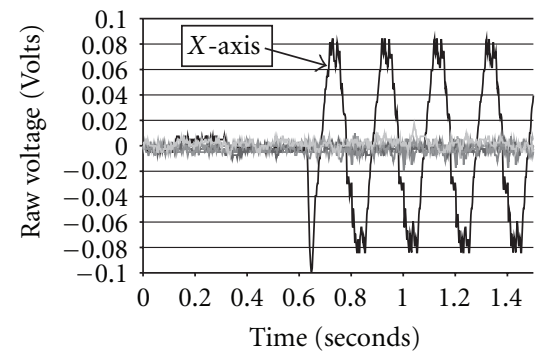

(a)

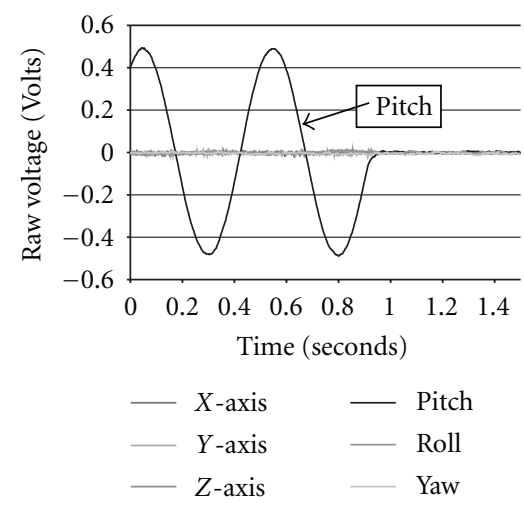

(d)

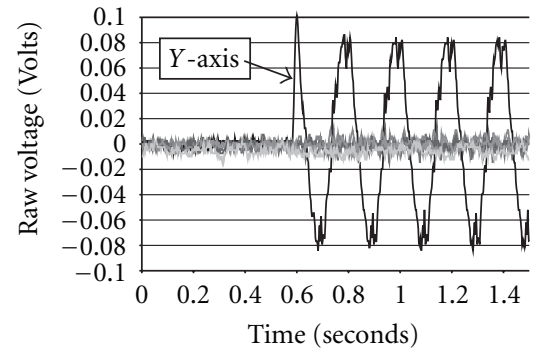

(b)

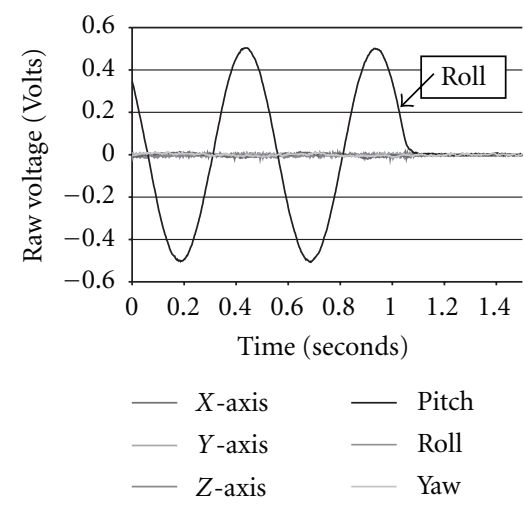

(e)

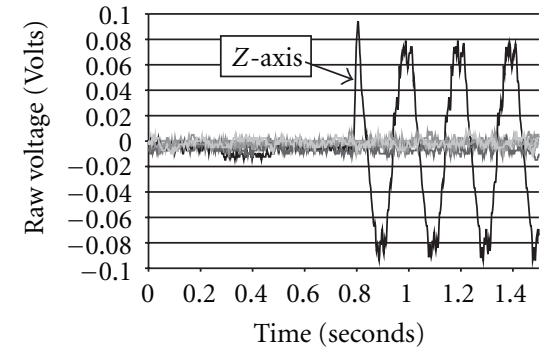

(c)

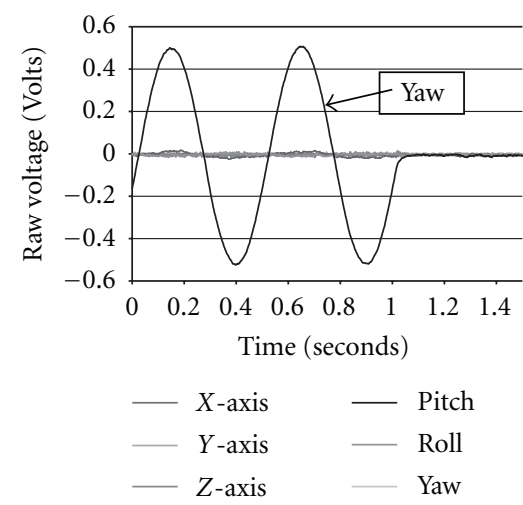

(f)

FIGURE 6: A sample of raw voltages recorded for single axis excitement with maximum and minimum translational acceleration amplitudes of $\pm 4 \mathrm{~m} / \mathrm{s}^{2}$ at $5 \mathrm{~Hz}$ or maximum and minimum angular velocity amplitudes of $\pm 40^{\circ} / \mathrm{s}$ at $2 \mathrm{~Hz}$; minimal cross-talk is apparent.

was minimized. The calibration data determined at LGs were applied to the SPT data, and compared to the VICON data with good results. It was shown in Figure 5 that the VICON and SPT accelerations are aligned, and the results in Table 5 indicate that the calibration determined at the LGs was successfully applied to HG accelerations.

The findings of this study in the HG portion compare with previous literature which found the difference between the VICON measurements and accelerometer data was less than $5 \%$ absolute error for displacements greater than $1 \mathrm{~mm}$ for frequencies between 3 and $30 \mathrm{~Hz}$ [11]. This error value does not seem unreasonable, as they are extremely small and fast motions (i.e., $1 \mathrm{~mm}$ at $30 \mathrm{~Hz}$ ). It is also possible that the errors reported by Jack et al. [11] may have resulted from the double differentiation process [14].

The purpose of the work was to provide a simple and reasonably fast calibration procedure and the procedure presented satisfied both constraints. In the future, a multiple resolution cross-correlation technique [15] could be used to automate data alignment which would streamline the alignment portion of the procedure. Other studies which have utilized a robot to calibrate accelerometers have relied on complex mathematical analyses. Renk et al. [16] developed a kinematic model which could be used by a 6-DOF robot arm to calibrate accelerometers. Kinematic model development required detailed math, whereas the PRSCO 6-DOF robot utilized in the current study requires simple sinusoidal equations and integration techniques. Others have reported instances where $6[17]$ or $9[17,18]$ linear accelerometer sensors were used to quantify 6-DOF movements in the field of biomechanics kinematic measurements. The calibration of these sensors requires the use of more complex mathematics than those required by the sensor and calibration procedure presented in this paper. The method outlined in the current paper can be used for WBV transducer calibration, as well as for almost any sensor measuring translational or rotational displacements, velocities, accelerations, or jerk. The method has been used successfully by our research group to quantify 6-DOF OSI and chassis accelerations in forestry skidders $[19,20]$.

In addition to the ease with which this calibration can be completed, the incorporation of the VTM for verification allows for comparisons between the sensor and VICON at various frequencies and displacements that exceed the PRSCO robot's capabilities. The inclusion of a dynamic calibration allows for more data points to be added to the data set. The increased number of data points likely increased the accuracy of the calibration equation compared to a simple static calibration procedure alone. In this case, the calibration verification was quick with more emphasis placed on the relationship between the VICON double differentiated displacements and the SPT accelerations. The HG data verification was included to confirm that the calibration determined easily at LG values did hold throughout the expected field testing range.

The methods outlined in this paper required little time, approximately three hours for the LG and five for the HG calibrations, including VICON Setup and calibration. The data 
processing took approximately 15 hours. Thus, a comprehensive calibration using a wide range of accelerations and angular rotations for a 6-DOF motion sensor may be performed in a reasonable amount of time by using the methods outlined in this paper.

\section{Conclusions}

This study described a method for calibrating multi-DOF sensors. The sensor calibrated in this study employed linear $(Y=m X)$ calibration equations resulting in $r^{2}$ values greater than 0.97 for maximum and minimum acceleration amplitudes of up to $\pm 8 \mathrm{~m} / \mathrm{s}^{2}$ and maximum and minimum velocity amplitudes up to $\pm 100^{\circ} / \mathrm{s}$. These calibrations in the translational axes were shown to hold for accelerations up to approximately $\pm 4 \mathrm{~g}$. Thus, procedures calibrated the SPT sensor through the expected range for 6-DOF WBV field measurements.

\section{References}

[1] Y. Marjanen and N. J. Mansfield, "Relative contribution of translational and rotational vibration to discomfort," Industrial Health, vol. 48, no. 5, pp. 519-529, 2010.

[2] M. J. Griffin, The Handbook of Human Vibration, Academic Press, London, UK, 1990.

[3] ISO, "ISO 2631-1: Mechanical vibration and shockevaluation of human exposure to whole-body vibrationpart 1," General requirements. International Organization for Standardization, 1997.

[4] British Standards Institution, "Measurement and evaluation of human exposure to whole-body mechanical vibration and repeated shock," BS 6841, 1987.

[5] C. M. Nelson and P. F. Brereton, "The European vibration directive," Industrial Health, vol. 43, no. 3, pp. 472-479, 2005.

[6] J.-M. Golsse and P. A. Hope, "Analysis of whole-body vibration levels during skidding," Tech. Rep. TR-77, Forest Engineering Research Institute of Canada (FERIC), 1987.

[7] S. Milosavljevic, D. I. Mcbride, N. Bagheri et al., "Exposure to whole-body vibration and mechanical shock: a field study of quad bike use in agriculture," Annals of Occupational Hygiene, vol. 55, no. 3, pp. 286-295, 2011.

[8] J.-M. Golsse, "Analysis of whole-body vibration levels during site preparation," Tech. Rep. TR-90, Forest Engineering Research Institute of Canada (FERIC), 1989.

[9] P. E. Boileau and S. Rakheja, "Vibration attenuation performance of suspension seats for off-road forestry vehicles," International Journal of Industrial Ergonomics, vol. 5, no. 3, pp. 275-291, 1990.

[10] S. Kumar, "Vibration in operating heavy haul trucks in overburden mining," Applied Ergonomics, vol. 35, no. 6, pp. 509-520, 2004.

[11] R. Jack, M. Oliver, and G. Hayward, "Validation of the VICON $^{\mathrm{TM}} 460$ motion capture system for whole-body vibration acceleration determination," in Proceedings of the 20th Congress of the International Society of Biomechanics and 29th Annual Meeting of the American Society of Biomechanics, Cleveland, Ohio, USA, July-August 2005.

[12] B. M. Nigg, G. K. Cole, and I. C. Wright, "Optical methods," in Biomechanics of the Musculo-Skeletal System, B. M. Nigg and W. Herzog, Eds., pp. 362-391, John Wiley \& Sons, New York, NY, USA, 3rd edition, 2007.
[13] L. Chiari, U. Della Croce, A. Leardini, and A. Cappozzo, "Human movement analysis using stereophotogrammetrypart 2: instrumental errors," Gait and Posture, vol. 21, no. 2, pp. 197-211, 2005.

[14] G. E. Robertson and G. E. Caldwell, "Planar kinematics," in Research Methods in Biomechanics, G. E. Robertson, G. E. Caldwell, J. Hamill, G. Kamen, and S. N. Whittlesey, Eds., Human Kinetics, Windsor, Canada, 2004.

[15] R. J. Jack, M. Oliver, R. Dony, and J. P. Dickey, "The use of multiple resolution cross-correlations to align simultaneously collected whole-body vibration data," Journal of Low Frequency Noise Vibration and Active Control, vol. 27, no. 2, pp. 121-133, 2008.

[16] E. L. Renk, M. Rizzo, W. Collins, F. Lee, and D. S. Bernstein, "Calibrating a triaxial accelerometer-magnetometer: using robotic actuation for sensor reorientation during data collection," IEEE Control Systems Magazine, vol. 25, no. 6, pp. 86-95, 2005.

[17] A. J. Padgaonkar, K. W. Krieger, and A. I. King, "Measurement of angular acceleration of a rigid body using linear accelerometers," Journal of Applied Mechanics, vol. 42, no. 3, pp. 552-556, 1975.

[18] N. K. Mital and A. I. King, "Computation of rigid-body rotation in three-dimensional space from body-fixed linear acceleration measurements," Journal of Applied Mechanics, vol. 46, no. 4, pp. 925-930, 1979.

[19] S. Cation, R. Jack, M. Oliver, J. P. Dickey, and N. K. LeeShee, "Six degree of freedom whole-body vibration during forestry skidder operations," International Journal of Industrial Ergonomics, vol. 38, no. 9-10, pp. 739-757, 2008.

[20] R. J. Jack, M. Oliver, J. P. Dickey, S. Cation, G. Hayward, and N. Lee-Shee, "Six-degree-of-freedom whole-body vibration exposure levels during routine skidder operations," Ergonomics, vol. 53, no. 5, pp. 696-715, 2010. 

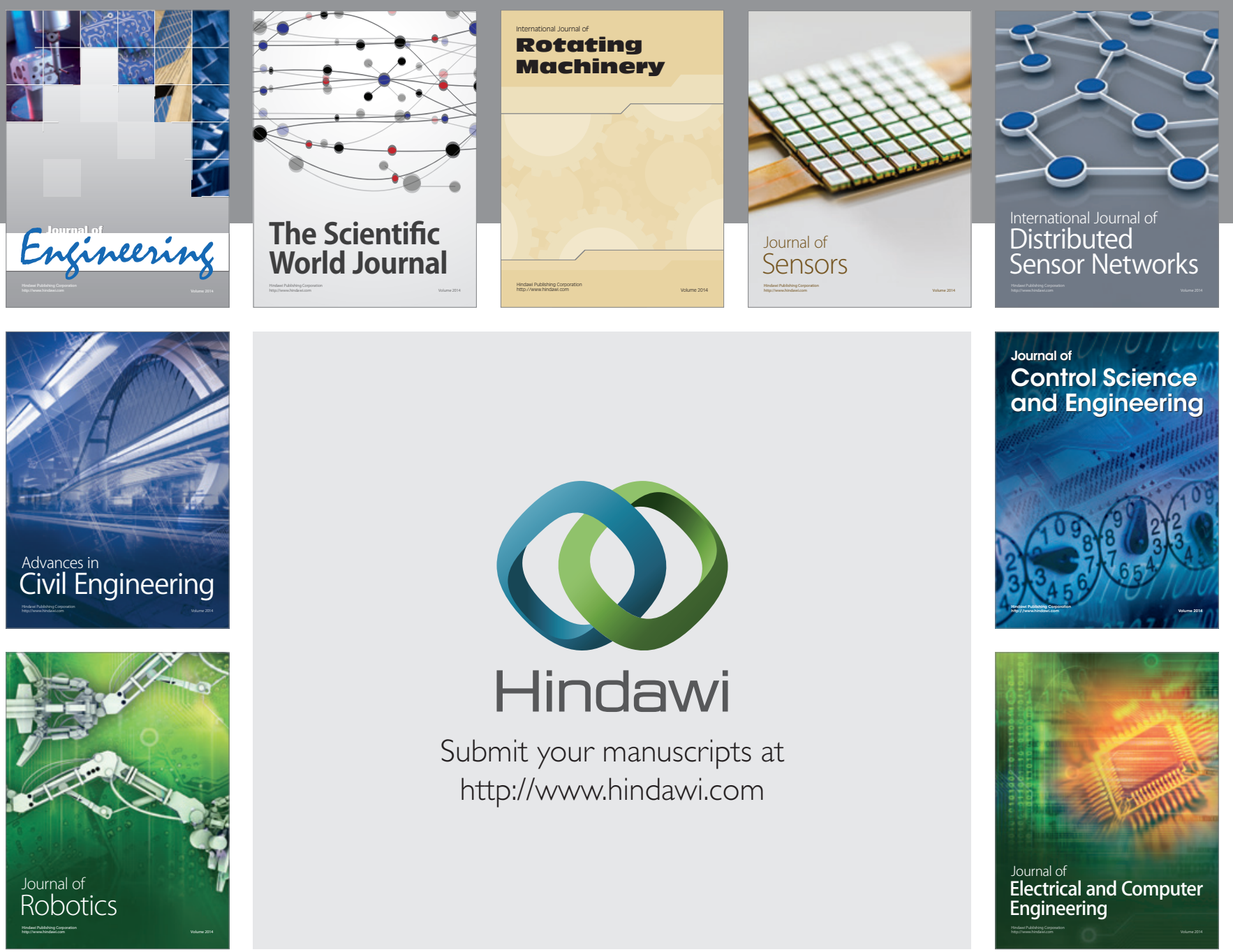

Submit your manuscripts at

http://www.hindawi.com
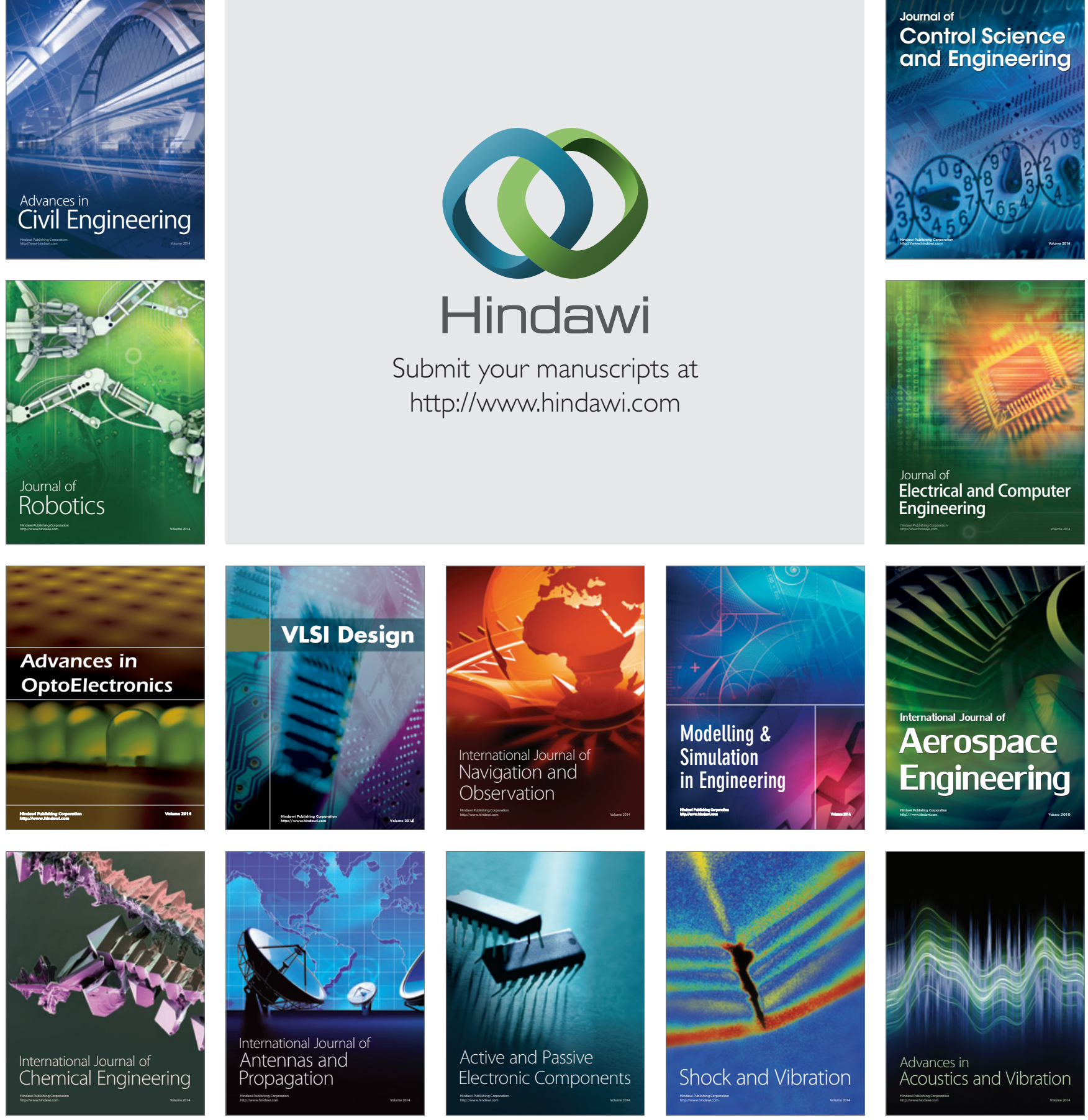